\title{
Feasibility of Group Visits for Advance Care Planning Among Patients with Heart Failure and Their Caregivers
}

\author{
Julia I. Bandini, PhD, Alexis Coulourides Kogan, PhD, Bonnie Olsen, PhD, \\ Jessica Phillips, MS, Rebecca L. Sudore, MD, David B. Bekelman, MD, MPH, and \\ Sangeeta C. Abluwalia, PhD
}

Background: Group visits have the potential to help patients identify their health care values and engage in the emotionally and cognitively challenging task of advance care planning (ACP) in a resource-efficient manner by providing a forum for social learning and social support.

Objective: To evaluate the feasibility and acceptability of disease-specific group visits for patients with heart failure and their caregivers.

Design: Feasibility trial of a 90-minute group visit held for 10 separate groups and led by a trained facilitator using the video-based PREPARE for Your Care ACP tool.

Setting:/Subjects: Older adults with recent hospitalization for heart failure $(n=36$; median age, 74 years) and their caregivers $(n=21)$.

Measurements: Pre- and post-visit surveys and a postvisit telephone interview assessing perceived value and acceptability; structured nonparticipant observations to assess process and feasibility.

Results: Mean scores from the postgroup visit evaluation showed that participants reported that they felt comfortable discussing ACP in a group (4.59), understood the information covered (4.70), and were able to identify and clarify their health care values (4.43). Interview and observation data demonstrated that participants were able to identify and clarify their preferences by listening and learning from a diverse range of perspectives in the group and that the disease-focused nature of the group visit created a supportive space for participants to share their experiences.

Conclusions: Disease-focused ACP group visits were feasible to conduct and acceptable to participants, underscoring their value as an efficient intervention to engage patients and caregivers in the otherwise time- and resource-intensive task of ACP. (J Am Board Fam Med 2021;34:171-180.)

Keywords: Advance Care Planning, Aging, Caregivers, Clinical Decision-Making, Decision Making, Feasibility Studies, Geriatrics, Heart Failure, Qualitative Research, Social Learning, Social Support, Surveys and Questionnaires, Terminal Care

\section{Introduction}

Advance care planning (ACP) is the process by which individuals think about, discuss, and communicate

This article was externally peer reviewed.

Submitted 1 May 2020; revised 8 September 2020; accepted 10 September 2020.

From RAND Corporation, Boston, MA (JIB); Department of Family Medicine and Geriatrics, Keck School of Medicine, University of Southern California, Alhambra, CA (ACK, BO); RAND Corporation, Santa Monica, CA (JP, SCA); Dep-artment of Medicine, Division of Geriatrics, University of California-San Francisco (RLS); San Francisco VA Medical Center, San Francisco, CA (RLS); Department of Medicine, Eastern Colorado VA Health Care System, Aurora, CO (DBB); Division of General Internal Medicine, University of Colorado School of Medicine at the Anschutz Medical Campus, Aurora, CO (DBB)

Funding: This study was funded by the National Institute on Aging of the National Institutes of Health under Grant Number R21-AG053716. RLS is funded in part by the their values and preferences for future care ${ }^{1,2}$ with the goals of achieving patient-centered and goalconcordant medical care. ${ }^{3}$ ACP is critically important in the context of life-limiting illness, particularly for older adults with heart failure who face a highly uncertain and variable trajectory with complex treatment choices to make. Unfortuna-tely, ACP continues to be limited and initiated late in the course of heart failure. Barriers to timely and effective ACP in this population include uncertainty about optimal

National Institute on Aging, National Institutes of Health (K24AG054415).

Conflict of interest: None.

Corresponding author: Sangeeta C. Ahluwalia, PhD, RAND Corporation, 1776 Main Street, Santa Monica, CA 90401 (E-mail: sahluwal@rand.org). 
timing of these conversations and reluctance to engage in emotionally challenging topics. ${ }^{4}$ Patients may have difficulty articulating their values and translating them into concrete preferences and choices regarding future decisions in the context of the uncertainty inherent to heart failure. ${ }^{5-8}$ Importantly, physicians lack the time and resources to engage in effective values communication during clinic visits ${ }^{4-8}$ and miss patient-initiated opportunities to engage in this process. ${ }^{\text {? }}$

Group visits for ACP, where several patients and/ or caregivers meet together with a single clinician or trained facilitator, are a scalable and resource-efficient health system intervention that may help to overcome some of these barriers. The group visit structure offers a forum where patients can learn from others' experiences, preferences, and choices by helping them to concretize their own health care values. Patients also have the opportunity to consider who they may choose and how much flexibility to give a surrogate decision maker. Group visits can provide important social support by encouraging patients to consider and engage in a typically difficult topic $^{10-12}$ and by motivating patients and their caregivers through social persuasion and action cues. ${ }^{12,13}$ For caregivers in particular, group visits have the potential to establish shared understanding regarding the patient's values and goals for care, and consequently increase caregiver confidence in proxy decision making. However, there are some limitations to the group visit model, such as for patients who are uncomfortable in a group setting or cognitively impaired. ${ }^{11}$ In addition, little is known about longerterm effectiveness of group visits at improving health outcomes. $^{14}$

A small but growing body of research has begun to explore the feasibility of ACP group visits for older adults in general, ${ }^{15,16}$ but little is known about the feasibility and utility of disease-specific group visits for ACP. Disease-focused visits may engender greater social connection and engagement by participants, more concrete ACP actions, and better understanding of the relevance of ACP in the context of a specific disease trajectory. Disease-focused ACP group visits may help a patient establish their values and goals for health care in the context of their illness, thereby supporting more effective future conversations with treating providers tailored to individual risk, prognosis, and salient treatment decisions.

Heart failure is prevalent among roughly one third of primary care patients over age 65 years and is associated with multiple comorbidities, ${ }^{17}$ underscoring the need to engage in ACP with heart failure patients in primary care settings. ACP group visits for heart failure patients could be a powerful and effective tool to help patients and their caregivers begin to engage in ACP. Prior work has shown that group visits for medical management of heart failure have resulted in increases in self care after 6 months, ${ }^{18}$ patient satisfaction, ${ }^{19}$ and overall knowledge of heart failure. ${ }^{20}$ Further, given the similarities in trajectory and ACP needs to other noncancer chronic conditions such as lung and kidney disease, ACP group visits for heart failure patients could provide a blueprint for other diseasefocused ACP group visits. In this study, we sought to evaluate the feasibility and acceptability of disease-specific group visits among patients with heart failure and their caregivers.

\section{Methods \\ Overview}

This study was part of a larger trial of ACP group visits for heart failure patients and their caregivers conducted at a large academic medical center in a major metropolitan area. We present findings related to the feasibility and acceptability of the group visits here; findings related to the preliminary impact of the group visits on ACP will be reported separately. We sought to understand the extent to which heart failure patients and their caregivers attend and participate in a group visit and the extent to which participants feel comfortable sharing health care experiences and discussing difficult topics in a group setting. We also wanted to understand which topics may be most appropriate and helpful for a heart failure-focused group visit and to explore the impact of different group sizes on discussions and social connection within the groups. We included caregivers in the group visits because of the important role caregivers play in health care decision making. All study procedures were reviewed and approved by institutional review boards.

\section{Participants}

We identified potential participants in two ways: 1) using administrative data, we identified patients aged $\geq 65$ years with at least 1 heart failure hospitalization (based on at least 1 of the following ICD-10 codes in the first 3 discharge diagnosis positions: 
I50.1, I50.20, I50.21, I50.22, I50.23, I50.30, I50.31, I50.32, I50.33, I50.40, I50.41, I50.42, I50.43, I50.9) within a 12-month period from the date of the data pull; and 2) project staff met with providers in the Advanced Heart Failure Clinic and Department of Family Medicine to describe the study and purpose of the group visits and encourage patient referrals to the study team. We also left project information sheets in the waiting rooms of the Cardiology Department and Family Medicine/Geriatrics Clinics. For all patients identified for possible inclusion in the study, we obtained demographic information necessary for initial contact and to confirm eligibility (age, mailing address, telephone number, number of hospitalizations, name of primary provider) from the medical record.

\section{Recruitment}

For patients identified through administrative data, we first contacted their primary provider via e-mail to request permission to contact their patient(s) regarding the study. We then mailed approved patients a study packet with information on the group visit, including a stamped postcard for the recipient to either indicate interest or decline participation and further contact. Study recruiters contacted by phone all patients who indicated interest via the returned postcard and those who did not respond within 1 week of mailing. On reaching the patient by phone, the study recruiters provided information on the group visit and if the patient was interested, administered a short cognitive screener, ${ }^{21}$ and scheduled the individual into a group visit. Patients were contacted up to three times before being removed from our list. Patients who self referred or were directly referred to the study by their provider were contacted by phone to schedule the group visit.

For all patients reached by telephone, study staff asked patients if they had someone who usually helped them make health care decisions and who may be interested in the study. If so, we attempted to speak to that person on the same call or obtained contact information to call later. Caregivers were administered the same screener and offered the same honorarium for participating. Caregivers could sign up to attend the group visit with the patient or on their own (ie, even if the patient did not want to or could not participate). We did not require patient permission for a caregiver to attend.

\section{Group Visit Intervention}

Participants were invited to attend 1 of 10 group visits offered on weekdays at varying times during the morning and afternoon hours. Group visits were 1.5 hours long, held in a conference room on the medical campus, and were led by a facilitator specifically trained in the group visit curriculum, with either a clinical psychology or health services background. The primary focus of the group visit was to help participants think about and clarify their health care values and preferences through structured group discussion and interactive exercises. All activities were guided by the evidence-based PREPARE for Your Care intervention (https://prepareforyourcare. org), which is an interactive, Web-based intervention with how-to video stories that is grounded in social cognitive theory and aimed at empowering patients to identify what matters most to them in their health care and moving them along the stages of ACP. ${ }^{22}$ PREPARE has been shown to significantly increase engagement in ACP and was rated easy to use among diverse older adults. ${ }^{23-25}$ The interactive Web version has been modified to be a video-based intervention for use during group visits. ${ }^{26}$ A PREPARE companion workbook with values clarification exercises and culturally relevant discussion questions to encourage structured discussions during group visits was provided to all participants. For the purpose of the current group visits we focused on the PREPARE steps most informative of values clarification and communication: "deciding what matters most," "choosing flexibility for your decision maker," and "telling others about your wishes." As time permitted or as the group discussion indicated, we also addressed the PREPARE steps of "how to ask doctors the right questions" and "choosing a medical decision maker." These steps were not proactively incorporated into the curriculum as they rely on individualized circumstances that might not be advantageous to explore in a group setting. Discussion on each step lasted between 15 and 25 minutes.

\section{Data Collection and Analyses}

We tracked recruitment and retention information, including number of eligible patients, number of patients who declined participation and reasons for declining, and number of patients and caregivers who attended a group visit. We characterized group composition based on participant type (patient or caregiver) as well as gender. Using closed-ended surveys, we collected 
participant demographics and feedback on the group visit, based on questions previously used to evaluate an advance directive intervention. ${ }^{27} \mathrm{We}$ gathered information on group dynamics and participant interactions using a structured, nonparticipant observational protocol during group visits. ${ }^{28}$ The protocol focused on how participants responded to questions posed by the facilitator, engaged with others in the group, and their emotional reactions to challenging topics. Within three days of attending a group visit, participants were contacted for a telephone interview conducted by a trained qualitative researcher, using a semistructured protocol covering what was most helpful to the participant, what was challenging about the group visit, perceptions about the educational tools (eg, video clips, workbook exercises), the extent to which the participant felt better prepared to engage in ACP, and additional topics the participant would have wanted to address in the visit. The interviewer took detailed notes during the interview.

All recruitment and survey data were descriptively analyzed. Qualitative data, including the follow-up interviews and observational data, were analyzed using a directed content analysis. ${ }^{29}$ Interview data were coded initially by interview question by one researcher and discussed with two other researchers. New and modified themes were developed throughout the analysis, and the detailed notes from each interview were recoded following the development of new themes.

\section{Results \\ Recruitment and Attendance}

Results from our recruitment process are shown in Figure 1. Of 444 eligible patients identified using administrative data, 27 were declined study participation by their primary provider. Although reasons for declining were infrequently provided by providers, 4 patients were declined because they were deceased at the time of contact. Of the 417 patients to whom we mailed the study packet, 10 patients declined participation by mailing back the enclosed postcard. We contacted by phone the remaining 407 patients, as well as another 12 patients who self referred or were directly referred by a provider, to assess interest and participation and schedule a group visit if applicable. Of these 419 identified patients contacted by phone, 183 (44\%) were unreachable within 3 phone attempts, and 89 (21\%) declined participation. An additional 28 patients $(6.7 \%)$ were deceased at the time of our phone call, another $20(5 \%)$ did not speak English, and 17 (4\%) were determined to be

Figure 1. Recruitment process and outcomes. Abbreviation: PCP, Primary care physician.

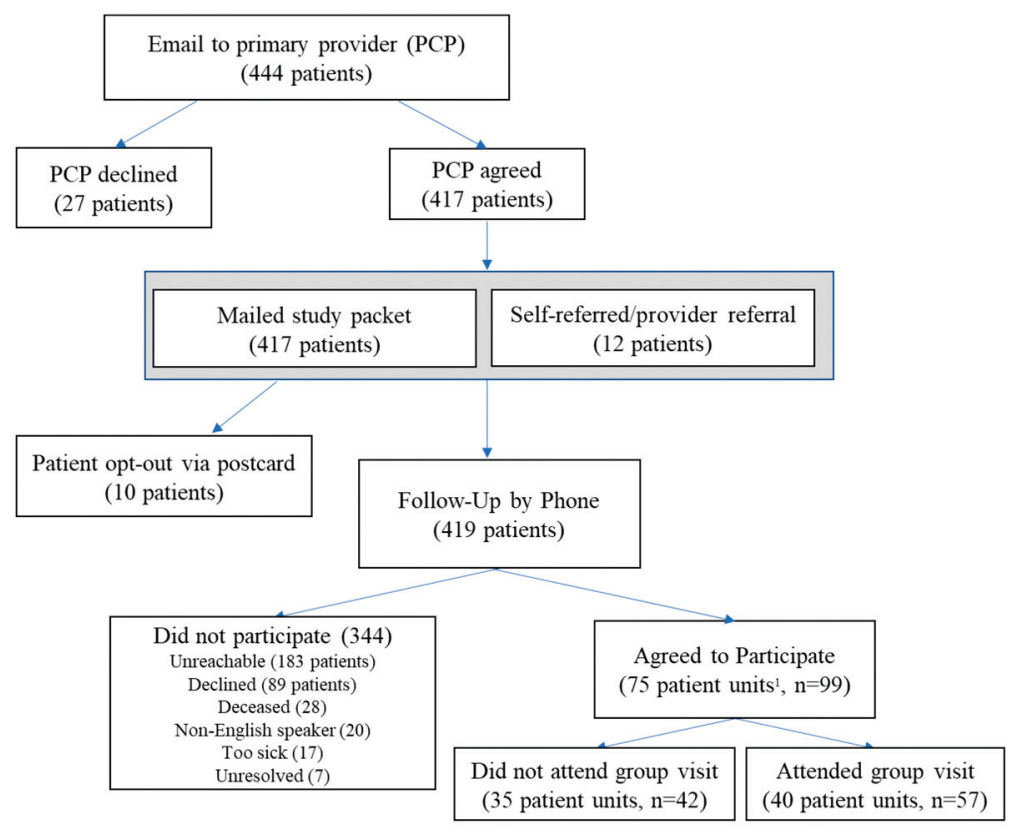

${ }^{1}$ Patient unit refers to the patient and any caregivers from the same family or group 
Table 1. Reasons for Not Attending Group Visit

\begin{tabular}{lcc}
\hline $\begin{array}{l}\text { Reasons for not attending group visit } \\
\text { (35 patient units, } \mathrm{n}=42)\end{array}$ & $\begin{array}{c}\text { Patient } \\
\text { Units }\end{array}$ & \begin{tabular}{c} 
Individuals \\
\hline No show
\end{tabular} \\
Never scheduled/censored* & 16 & 21 \\
Cancelled, no reason given & 14 & 14 \\
Language difficulty & 2 & 4 \\
Became too sick to attend & 2 & 2 \\
Total & 1 & 1 \\
\hline
\end{tabular}

*Project ended before they could be scheduled into a group visit.

too ill to attend an in-person group visit. There were 7 patients who were censored (see Table 1).

The remaining 75 (17.9\%) identified-patients agreed to participate, with or without a caregiver, or in some cases a caregiver without the patient, resulting in 99 individuals who agreed to participate in a group visit. Of these 99 individuals, 57 attended a group visit. The remaining 42 individuals agreed to participate but ultimately did not attend a group visit (Table 2). The majority of these individuals $(\mathrm{n}=21$; $50 \%$ ) did not show up to their scheduled group visit and subsequently became unreachable; the other 14 individuals $(33 \%)$ were never able to be scheduled into a group visit before the study ending.

\section{Group Visit Composition and Size}

A total of 36 patients and 21 caregivers $(n=57)$ attended 1 of 10 available group visits held between July 2018 and February 2019 (Table 2). These 57 participants represented 40 unique patient/caregiver units; 22 patients attended alone, 4 caregivers attended alone (eg, because the patient was otherwise unable to attend), and 14 patient-caregiver units (11 dyads, 3 triads) attended together. Group visits ranged in size from 3 to 10 participants (2 groups had 3 participants; one group had 10 participants). Patient participants had a median age of 74 years (collected from administrative data), 18 $(50 \%)$ were women, and $26(72 \%)$ were white. Caregiver participants $(\mathrm{n}=21)$ were mostly female (81\%) and also mainly white (57\%). Caregiver participants included 8 adult children (all daughters), 8 spouses, 2 friends, 2 caregivers, and 1 grandchild.

\section{Survey Feedback}

Feedback from participants on the group visits was positive (Table 3). The majority of both patients and caregivers reported that they felt comfortable discussing a range of topics (patient mean, 4.59; caregiver mean, 4.7), they understood the information discussed (patient mean, 4.7; caregiver mean, 4.74), and that the right amount of information was provided (patient mean, 4.57; caregiver mean, 4.5). Patients and caregivers also reported that the visit helped them to clarify their values and goals for care (patient mean, 4.43; caregiver mean, 4.44) and that they learned about ACP (patient mean, 4.43; caregiver mean, 4.5).

\section{Participant Interviews}

Debrief interviews were conducted with 41 participants from 34 different patient units (29 patients, 12 caregivers) within 3 days of their group visit and had a mean length of 15.5 minutes. The remaining 16 individuals ( 7 patients, 9 caregivers) were unable to be reached for a follow-up interview. In general, participants described positive experiences with the group visit and spoke about how the nature of the group visit made a sensitive topic comfortable to discuss in a group setting. Participants found the different modes of learning tools including the video and the PREPARE guide to be helpful aspects of the curriculum.

Four elements of group dynamics that emerged from the interviews further highlight the benefits of these visits: 1 ) participants were able to identify and clarify their health care preferences by hearing the experiences and preferences of other patients with heart failure; 2) participants were better able to understand the disease experience from multiple perspectives because of the mixed composition; that is, caregivers and patients together and a mix of genders; 3 ) the diseasefocused nature of the visits created a safe space for

Table 2. Participant Demographics

\begin{tabular}{lccc}
\hline & $\begin{array}{c}\text { Patients } \\
(\mathrm{n}=36)\end{array}$ & $\begin{array}{c}\text { Caregivers } \\
(\mathrm{n}=21)\end{array}$ & $\begin{array}{c}\text { Total } \\
(\mathrm{n}=57)\end{array}$ \\
\hline Age, median* & 74 & N/A & N/A \\
Female, n (\%) & $18(50)$ & $17(81)$ & $35(61)$ \\
Race/ethnicity, n (\%) & & & \\
$\quad$ White & $26(72)$ & $12(57)$ & $38(67)$ \\
Black & $4(11)$ & $0(0)$ & $4(7)$ \\
Latino/Hispanic & $4(11)$ & $5(24)$ & $9(16)$ \\
Asian Pacific Islander & $0(0)$ & $1(5)$ & $1(2)$ \\
Other/multiethnic & $2(6)$ & $3(14)$ & $5(9)$ \\
Married & $16(44)$ & $14(67)$ & $30(53)$ \\
\hline
\end{tabular}

* Median based off of 31 patients (patient age missing for 5 patients). 


\begin{tabular}{lllll}
\hline & \multicolumn{2}{c}{ Patients $(\mathrm{n}=36)$} & \multicolumn{2}{c}{ Caregivers $(\mathrm{n}=21)$} \\
\cline { 2 - 5 } Participant Response & Mean & SD & Mean & SD \\
\hline I felt comfortable discussing the topics covered in today's group visit. & 4.59 & 0.76 & 4.7 & 0.47 \\
I understood what was discussed today. & 4.7 & 0.74 & 4.74 & 0.45 \\
The group visit provided the right amount of information. & 4.57 & 0.80 & 4.5 & 0.61 \\
The group visit helped me to identify and clarify my healthcare values. & 4.43 & 0.83 & 4.44 & 0.70 \\
The group visit helped me learn more about advance care planning. & 4.43 & 0.89 & 4.5 & 0.76 \\
The time allotted for the group visit was adequate. & 4.24 & 0.86 & 4.5 & 0.51 \\
\hline
\end{tabular}

SD, standard deviation.

participants to share their disease experience and contemplate values and goals in the context of heart failure; and 4) the visits were an important source of social support, group identification/ cohesion, and camaraderie (Table 4).

\section{Structured Non-Participant Observations}

Observations conducted during the group visits showed that participants appeared comfortable and that the discussions flowed well with participants reflecting, sharing, and validating others' experiences. The two larger groups (over 8 participants) had more cross-talk among participants, which on the one hand seemed to encourage further sharing within the group, but on the other hand required the facilitator to intervene more often and redirect conversations. The three smaller groups (composed of 3 participants) seemed to have a longer "warmup" period where participants appeared comfortable and relaxed enough to engage and share experiences, especially when composed of only a patient/ caregiver dyad and another patient. In addition, less diversity of experiences was shared in smaller groups than larger groups. While not universal, many participants mentioned that they had previously completed an advance directive. Several participants also spontaneously and voluntarily shared personal and challenging experiences with heart failure in addition to other comorbid conditions (ie, diabetes, kidney disease, cancer), noting multiple hospitalizations, surgeries, and complex medical decisions they had previously faced. Across all groups, some content was raised that required redirecting and did not seem conducive to group discussion given their sensitive or personal nature. These topics included physician-assisted suicide, individual health concerns, individual religious beliefs, financial issues, and specifics complaints about the health care system.

\section{Discussion}

Our findings suggest that a disease-specific group visit model is a feasible and acceptable approach for engaging heart failure patients and their caregivers in ACP. Despite study recruitment challenges, the majority of heart failure patients and caregivers who attended the group visit agreed or strongly agreed that they felt comfortable discussing the topics, understood the information presented, and that the group visit helped them learn about ACP. Older adults with heart failure were willing to share personal experiences about their preferences for medical care and spoke broadly of a range of health care experiences with heart failure and other comorbid conditions in the context of ACP. The combination of patients and caregivers, either as dyads from the same family or as separate attendees, allowed for reflexivity and enabled participants to hear a range of experiences with heart failure and views around planning for future care from different perspectives.

While prior work has explored group visits for ACP among the general older adult population, ${ }^{15,16}$ we focused on heart failure-a highly prevalent life-limiting illness affecting more than 6 million Americans ${ }^{17}$ - to purposefully identify patients who might share similar health care experiences and concerns informative of ACP. This allowed us to contextualize the ACP process within the uncertainty of heart failure and the complex treatment decisions (eg, Implantable Cardioverter-Defibrillator or Left Ventricular Assist Device implantation) participants likely had already made or would be expected to make. 
Table 4. Group Dynamics Themes from Follow-Up Interviews

\begin{tabular}{|c|c|}
\hline Group Dynamics Theme & Quote \\
\hline $\begin{array}{l}\text { 1. Participants leveraged others' experiences to identify and } \\
\text { clarify their own preferences. }\end{array}$ & $\begin{array}{l}\text { "I think being in a group was helpful because you can get ideas } \\
\text { from others about tricks or ways to talk about this and how } \\
\text { others do it-getting information from other people was } \\
\text { helpful. Piece together others' strategies for talking about these } \\
\text { things and take what is helpful for me." (Patient) } \\
\text { "It was interesting and you learn from what other people have } \\
\text { done and gone through. I find that more interesting and more } \\
\text { valuable to learn more about other people's experiences." } \\
\text { (Patient) } \\
\text { "I was able to hear other people's stories and that was a value to } \\
\text { me. I was not intimidated in the group visit at all. I find it better } \\
\text { to be in a group visit like that than to do it on your own." } \\
\text { (Patient) }\end{array}$ \\
\hline
\end{tabular}

2. There was value in mixed compositions of the groups to understand alternative perspectives (i.e. patients and caregivers together; a mix of genders).
3. Disease-focused nature and support group aspect provided participants with a safe space to share difficult experiences as well as their preferences and values.

4. Group visit facilitated camaraderie among participants
"I thought about what my family is going through with me with the heart condition, my family and my friends. You see the other side of the coin!" (Patient)

"And hearing others' experiences put a face to it. It was good to hear people speak because I haven't had that experience in my life." (Caregiver)

"I think it actually helped [to be with others] because without having your spouse there who feels totally different than I do, it helps to hear other people who think that. I think it's very helpful." (Patient)

"They were all women, except mostly me, it would have been nice if I could relate to other men." (Patient)

"I just think being around other people who have had similar experiences is always helpful even if it might be an uncomfortable topic." (Patient)

"Well being one of the heart failure people, it was good. (laughs) [What made it good?] It was kind of close to a support group knowing other people there have the same issues." (Patient)

"I do think it was helpful for a group setting. It's not an easy thing to talk about in our culture. It's not something you talk about during a meal. It's kind of hard for the kids. You don't want to talk about it. It's never really a good time to bring it up even though it's always in the back of people's minds. It's educational, kind of thing-you go there and learn about it. It would be easier for people to swallow that in a group setting. Yeah I think it's a good way of doing it." (Caregiver)

"[What was most helpful?] Actually it was the input from the other people there-we kept thinking we're on our own problems-but I think other people have the same problems." (Caregiver)

"She [facilitator] was really good. She was very helpful. She made us feel very friendly with each other. And in one hour or a couple of others, we felt like we were actually friends. And we started talking and feeling good about each other. So she made it happen." (Patient)

"It stimulated a lot of conversation. When I was leaving people were talking at the elevators. But I liked what I saw when it was over." (Patient)

"[What did you like best?] Probably the interaction among all the people. We actually got some side benefits from it from contacts to be made from people who are having the same problems as my husband." (Caregiver)
While participants often shared health care experiences related to comorbid conditions, the foundation of shared experience with heart failure helped participants establish an initial bond that engendered a broader discussion regarding health care values. As such, disease-focused group visits may be one strategy for initiating ACP discussions by creating social connections through shared experiences. Moreover, the prevalence of heart failure and the similarity of its trajectory to other noncancer conditions, such as chronic obstructive pulmonary disease (COPD) or kidney disease, 
suggests the relevance and value of diseasefocused group visits to a large population of patients cared for in primary care settings.

Group structure and dynamics during a diseasespecific group visit are important for facilitating a robust conversation and safe space for discussion. We did not have a target number for group size, but rather explored how different sizes and composition (ie, patient and caregivers) facilitated discussion and social learning. Based on nonparticipant observations, a well-balanced group of 6 to 8 participants with a mix of patients and caregivers allowed for productive discussion and encouraged sharing of a diverse range of experiences. The combination of both patients and caregivers in the group visit allowed for sharing of opposing experiences and facilitated core aspects of social learning such as perspective-taking, reflexivity, and empathy. This social interaction and shared storytelling played an important role in the ability for participants to consider their own values and wishes.

In this study, we offered a single ACP group visit to heart failure patients and caregivers. An ACP group visit could serve as the cornerstone of an iterative ACP process, occurring early in the illness trajectory and aimed at establishing a baseline understanding of values and priming patients and surrogates for more tailored subsequent patient-provider discussions focused on specific treatment choices. Sentinel events along the course of an illness, such as a heart failure hospitalization could trigger follow-up group visits aimed at re-evaluating one's health care values. While the current study was a small feasibility trial, future work could explore the value of a group visit series and assess their longitudinal and cumulative impact on ACP outcomes.

On a health systems level, group visits are an organizationally efficient and highly rated model for ACP that could reduce costs associated with providers' time spent individually with patients and their caregivers discussing ACP, as noted in other group visit studies. ${ }^{15,16}$ While the Centers for Medicare and Medicaid Services now provide reimbursement for counseling in $\mathrm{ACP},{ }^{30}$ there are potential challenges for sustainability of the group visit model for ACP around adequate reimbursement for ACP discussions conducted in a group setting. ${ }^{15}$ Costs might be offset by the use of a nonphysician trained facilitator and long-term reductions in medical visits, and many health systems are already successfully sustaining group visits for other purposes. ${ }^{31,32}$
The timing of disease-specific group visits for ACP is particularly important for patients with heart failure. These patients have variable illness trajectories, creating challenges for finding a time when they are healthy enough to attend a group visit, yet with enough illness experience to effectively consider their health care values and goals. Some patients who attended the group visit were further along in their disease trajectory and able to draw from and share a variety of health care experiences and complex medical decisions that they had already faced, underscoring the social learning benefit of group visits. Furthermore, many of these patients were already well versed in the ACP process, potentially limiting the benefit they might derive from attending a group visit aimed at values clarification. It will be important for diseasefocused group visits in the future to ensure diversity of experience with both heart failure and ACP to maximize their utility to a wide range of patients and their caregivers.

We also do not have information on why patients declined participation in the group visit $(n=89)$. It is possible that patients may have declined participation because they already had an advance directive or, on the other hand, were not ready to engage in conversations around end-of-life care, as older patients may be at different stages of readiness to engage in $\mathrm{ACP} ;{ }^{22}$ however, we do not have information on advance direction completion or on stages of ACP readiness among those who declined. It is also possible that these individuals did not understand the scope or purpose of the group visit. Several patients and caregivers mentioned in the follow-up interviews that they did not know what to expect or had misperceptions around the content of the group visit before attending. Given low completion rates of advance directives in geriatric populations, ${ }^{33}$ recruitment efforts for disease-specific group visits for ACP may need further refinement for clarifying the purpose of the group visit and expectations.

\section{Limitations}

This study was conducted at a single site in the Southwestern region of the United States. We have limited data on the reasons why some patients declined participation in the group visit. Patients and caregivers who attended the group visit were mostly white, and caregivers were predominantly female. There is the potential for selection bias, as 
individuals in this study who chose to participate in a group visit may have been more likely to be open to sharing their values and preferences in a group setting. We also offered an honorarium for participation as to offset the time commitment associated with attending the group visit and participating in the debrief interview, which may have been an incentive for some to participate. Future work may consider feasibility and efficacy of a disease-specific group visit intervention for conditions other than heart failure and also for wider implementation across health care settings.

\section{Conclusion}

Disease-specific group visits for heart failure like those for the general geriatric population ${ }^{15,16}$ may serve as a feasible and efficient intervention to engage more patients at younger ages and their caregivers in the emotionally challenging and resource-intensive process of ACP.

We thank Pau Alonso Garcia-Bode, who provided data analytic support for this manuscript.

To see this article online, please go to: http://jabfm.org/content/ 34/1/171.full.

\section{References}

1. Sudore RL, Heyland DK, Lum HD, et al. Outcomes that define successful advance care planning: a Delphi Panel consensus. J Pain Symptom Manage 2018;55:245-255.

2. Sudore RL, Fried TR. Redefining the "planning" in advance care planning: preparing for end-of-life decision making. Ann Intern Med 2010;153:256-61.

3. Institute of Medicine; Committee on Approaching Death. Dying in America: improving quality and honoring individual preferences near the end of life. Washington, DC: The National Academies Press; 2015.

4. Tung EE, North F. Advance care planning in the primary care setting: a comparison of attending staff and resident barriers. Am J Hosp Palliat Care 2009;26:456-63.

5. Goodlin SJ, Quill TE, Arnold RM. Communication and decision-making about prognosis in heart failure care. J Card Fail 2008;14:106-13.

6. Selman L, Harding R, Beynon T, et al. Improving end-of-life care for patients with chronic heart failure: "let's hope it'll get better, when I know in my heart of hearts it won't." Heart 2007;93:963-7.

7. Harding R, Selman L, Beynon T, et al. Meeting the communication and information needs of chronic heart failure patients. J Pain Symptom Manage 2008;36:149-56.

8. Barclay S, Momen N, Case-Upton S, Kuhn I, Smith E. End-of-life care conversations with heart failure patients: a systematic literature review and narrative synthesis. Br J Gen Pract 2011;61:e49-e62.

9. Ahluwalia SC, Levin JR, Lorenz KA, Gordon HS. Missed opportunities for advance care planning communication during outpatient clinic visits. J Gen Intern Med 2012;27:445-51.

10. Beck A, Scott J, Williams P, et al. A randomized trial of group outpatient visits for chronically ill older HMO members: the Cooperative Health Care Clinic. J Am Geriatr Soc 1997;45:543-9.

11. Scott JC, Conner DA, Venohr I, et al. Effectiveness of a group outpatient visit model for chronically ill older health maintenance organization members: a 2-year randomized trial of the cooperative health care clinic. J Am Geriatr Soc 2004;52:1463-70.

12. Jaber R, Braksmajer A, Trilling JS. Group visits: a qualitative review of current research. J Am Board Fam Med 2006;19:276-90.

13. Lorig KR, Holman H. Self-management education: history, definition, outcomes, and mechanisms. Ann Behav Med 2003;26:1-7.

14. Housden L, Wong ST, Dawes M. Effectiveness of group medical visits for improving diabetes care: a systematic review and meta-analysis. CMAJ 2013;185:E635-E644.

15. Lum HD, Jones J, Matlock DD, et al. Advance care planning meets group medical visits: the feasibility of promoting conversations. Ann Fam Med 2016;14: 125-32.

16. Lum HD, Sudore RL, Matlock DD, et al. A group visit initiative improves advance care planning documentation among older adults in primary care. $\mathrm{J}$ Am Board Fam Med 2017;30:480-90.

17. Bosch L, Assmann P, de Grauw WJC, Schalk BWM, Biermans MCJ. Heart failure in primary care: prevalence related to age and comorbidity. Prim Health Care Res Dev 2019;20:e79-e79.

18. Lin A, Cavendish J, Boren D, Ofstad T, Seidensticker D. A pilot study: reports of benefits from a 6-month, multidisciplinary, shared medical appointment approach for heart failure patients. Mil Med 2008;173:1210-3.

19. Paul S, Yehle KS, Wood K, Wingate S, Steg B. Implementing shared medical appointments for heart failure patients in a community cardiology practice: a pilot study. Heart Lung 2013;42:456-61.

20. Yehle KS, Sands LP, Rhynders PA, Newton GD. The effect of shared medical visits on knowledge and self-care in patients with heart failure: a pilot study. Heart Lung 2009;38:25-33.

21. Pfeiffer E. A short portable mental status questionnaire for the assessment of organic brain deficit in elderly patients. J Am Geriatr Soc 1975;23:433-41. 
22. Fried TR, Redding CA, Robbins ML, Paiva A, O'Leary JR, Iannone L. Stages of change for the component behaviors of advance care planning. J Am Geriatr Soc 2010;58:2329-36.

23. Zapata C, Lum HD, Wistar E, Horton C, Sudore RL. Feasibility of a video-based advance care planning website to facilitate group visits among diverse adults from a safety-net health system. J Palliat Med 2018;21:853-6.

24. Sudore RL, Knight SJ, McMahan RD, et al. A novel website to prepare diverse older adults for decision making and advance care planning: a pilot study. J Pain Symptom Manage 2014;47: 674-86.

25. Lum HD, Barnes DE, Katen MT, Shi Y, Boscardin $\mathrm{J}$, Sudore RL. Improving a full range of advance care planning behavior change and action domains: The PREPARE randomized trial. J Pain Symptom Manag 2018;56:575-581.

26. Sudore RL, Boscardin J, Feuz MA, McMahan RD, Katen MT, Barnes DE. Effect of the PREPARE website vs an easy-to-read advance directive on advance care planning documentation and engagement among veterans: a randomized clinical trial. JAMA Intern Med 2017;177: 1102-9.
27. Sudore RL, Landefeld CS, Barnes DE, et al. An advance directive redesigned to meet the literacy level of most adults: a randomized trial. Patient Educ Couns 2007;69:165-95.

28. DeWalt KM, DeWalt BR, Wayland CB. Participant observation. Handbook Methods Cultur Anthropol 1998;259-300.

29. Hsieh HF, Shannon SE. Three approaches to qualitative content analysis. Qual Health Res 2005;15:1277-88.

30. Frequently Asked Questions about Billing the Physician Fee Schedule for Advance Care Planning Services Centers for Meicare and Medicaid Services Web site. 2016. Available from: https://www.cms. gov/Outreach-and-Education/Medicare-LearningNetwork-MLN/MLNProducts/Downloads/Advance CarePlanning.pdf. Accessed July 22, 2020.

31. Sikon A, Bronson DL. Shared medical appointments: challenges and opportunities. Ann Intern Med 2010;152:745-6.

32. Cherniack EP. The use of shared medical appointments in the care of the elderly. J Ambul Care Manage 2014;37:32-7.

33. Yadav KN, Gabler NB, Cooney E, et al. Approximately one in three US adults completes any type of advance directive for end-of-life care. Health Aff (Millwood) 2017;36:1244-51. 